\title{
Asymptotically Optimal Scenario-based Multi-objective Optimization for Distributed Generation Allocation and Sizing in Distribution Systems
}

\author{
Lizhen $\mathrm{Wu}^{1}$, Xusheng Yang ${ }^{1}, \mathrm{Hu} \mathrm{Zhou}^{2}$, Xiaohong Hao ${ }^{1}$ \\ ${ }^{1}$ College of Electrical and Information Engineering of Lanzhou University of \\ Technology, Lanzhou, Gansu,730050,China \\ ${ }^{2}$ Gansu Electric Power Corporation, Lanzhou, Gansu, 730030, China \\ wulzh@lut.cn
}

\begin{abstract}
Suitable location and optimal sizing are impact on voltage stability margin of the distributed system. It is important to accurately simulate the random output active power of Distributed Generation (DG). In order to model uncertainties of intermittent distributed generation and load, this paper proposes a multi-scenario tree model of windphotovoltaic-load using multiple scenarios technique based on the Wasserstein distance metrics, which generates asymptotically optimal scenario. And in this paper, a multiobjective optimizes control model with scenario tree is presented, which including objectives that are the total active power losses and the voltage deviations of the bus. Moreover, a new hybrid Honey Bee Mating Optimization and Particle Swarm Optimization (HBMO-PSO) algorithm is proposed to solved the problems. In the HBMOPSO algorithm, the mating process is corrected, which the PSO algorithm is combined with the HBMO algorithm to improve the performance of HBMO. Finally, a typical IEEE 33-bus distribution test system is used to investigate the feasibility and effectiveness of the proposed method. Simulation results illustrate the correctness and adaptability of the proposed model and the improved algorithm.
\end{abstract}

Keywords: Multi-objective optimization, Honey Bee Mating Optimization (HBMO), Distributed Generation (DG), Optimal Scenario, Voltage Profile

\section{Introduction}

Distributed energy resource (DER) is attracting more and more attentions due to the economic, technical and environmental problems of the conventional distribution systems [1]. However, high penetration of DG sources into the grid will bring new challenges for the efficient and safe power system operation, especially on the distribution networks. Using DG sources impose a different set of operating factors in distribution network such as voltage rise, reverse power flow, reduction of power losses and decreasing fault level, harmonic distortion and stability problems [2]. The goal of active distribution system is to allow safe penetration of DERs into current distribution systems and maintain the system stably and reliably [3]. The impacts of DG on various aspects of distribution system operation depend highly on location and size of DG. Location and sizing of DGs voltage stability, voltage quality and power loss in distribution network[4].

The allocation of DG units in distribution systems has been investigated in the literature from different perspectives. In Ref. [5], the impact of increasing DG penetration on system losses has been analyzed for different generating resources. In Ref. [6], an analytical approach is developed to minimize energy losses by optimal DG placement in a distribution system. The selection of the best places for installation and the preferable size of the DG units in large distribution systems is a complex combinatorial optimization 
problem .In Ref. [7] presented a methodology based on genetic algorithm (GA) is to minimize network losses with the consideration of system constraints such as reliability, voltage limits, and DG penetration. In Ref. [8] the objective function is optimization of voltage profile. All these mentioned objective function are single-objective and in this paper all of them are considered together as multi-objective. Various methods were used for solving optimization problem. In Ref. [9] dynamic ant colony search algorithms are used to achieve aims. In Ref. [10] employs an artificial bee colony algorithm to determine the optimal DG-unit's size, power factor, and location in order to minimize the total system real power loss. But they ignore the stochastic characteristics of DGs. The uncertainties of intermittent DG and load have adverse impacts on active distribution system. Recently, Some of the literature studied the stochastic characteristics of DGs and established the Probabilistic-Scenario Model[11-15].

Thus in this paper, accounts the uncertainties of load demand, wind speed and solar radiation by using probabilistic method. The asymptotically optimal scenario generated by using Wasserstein distance metrics, which simulate wind power and photovoltaic output power. According to the idea of scenario tree model, the multi-scenario tree models of wind-photovoltaic-load are established, that takes account the stochastic property of wind energy, photovoltaic and load. A multi-objective optimizes control model consider multiple scenarios tree is presented, which including objectives that are the total active power losses; the voltage deviations of the bus, and constrains of power flow equations, the substation capacity, node voltage magnitude and branch current, etc. Moreover, a new hybrid Honey Bee Mating Optimization and Particle Swarm Optimization (HBMO-PSO) algorithm is proposed to solved the Multi-objective Optimization Problems. Finally, taking the typical IEEE33 distribution network system as an example, the simulation results show that the optimal DG is beneficial to improve the distribution network voltage stability margin, reduce the loss of network system.

This paper is organized as follows: the multi-scenario tree models of windphotovoltaic-load are presented in Section II. The multi-objective model based on asymptotically optimal scenario for sitting and sizing of the DGs and its solution are discussed in Section III. In Section IV the feasibility of proposed approach is demonstrated and its performance is compared with other methods for IEEE 33-bus distribution test feeder. Section V is the conclusions.

\section{Multi-scenario Tree Models of Wind-Photovoltaic-Load}

\subsection{Asymptotically Optimal Scenario Generation based on Wasserstein Distance Metric}

Using a discrete probability distribution $\left(\left(\varsigma, \widetilde{G}_{i}\right), i=1,2 \ldots S\right)$ to represent the random variable uncertainty is called scenario simulation. The difference between the scenario model and the original model is the use of discrete probability distributions of $\tilde{G}$ to represent the original probability distribution $\mathrm{G}$. The space of probability measures (i.e. the so-called Wasserstein metric space). The probability measure that is closest to a given collection of input measures in the sense that it will minimize the (weighted) Wasserstein distance between itself and the inputs. The accuracy of asymptotic optimal scenario simulation method based on Wasserstein distance metric is better than the other distance function[14]. Assuming that the probability of edge distribution function is $x \sim G$ and discrete probability edge function is $\tilde{x} \sim \tilde{G}$, then their Wasserstein distance can be expressed as $d(G, \tilde{G})$.The basic idea of scenario simulation is assuming $\mathrm{G}$ known probability measure on RM, for a probability measure $\tilde{G}$ point set based on $\mathrm{S}$, make the distance metric $d(G, \tilde{G})$ smaller.

According to the ref.[14],the optimal single dimensional random variable quantile $Z_{s}$ 
solving equations by means of asymptotic strategy as follows:

$\int_{-\infty}^{Z_{s}} G^{1 /(1+p)}(x) d x=\frac{2 s-1}{2 S} \int_{-\infty}^{\infty} G^{1 /(1+p)}(x) d x$

Probability corresponding quantile $\mathrm{Z}_{\mathrm{n}}$ according to (2) - (4) can be calculated:

$p_{s}=\int_{\frac{z_{s}+z_{s-1}}{2}}^{\frac{z_{s}+z_{s+1}}{2}} f(x) d x, \quad s=2, \cdots, S-1$

$p_{0}=\int_{z_{0}}^{\frac{z_{s}+z_{s-1}}{2}} f(x) d x, s=2$

$p_{S}=\int_{\frac{z_{s}+z_{s+1}}{2}}^{z_{S}} f(x) d x, \quad s=S-1$

Where $Z_{\bar{\sigma}}=-\infty ; Z_{S}=+\infty$

\subsection{Asymptotically Optimal scenario of Wind Power and PV}

In this paper, the probability density functions (PDF) for generated wind power scenario are as follows ${ }^{[14,15]}$ :

$$
f_{w}(P)=\frac{k h v_{i n}}{P_{r} c}\left[\frac{\left(1+(h P) / P_{r}\right) v_{i n}}{c}\right]^{k-1} \exp \left\{-\left[\frac{\left(1+(h P) / P_{r}\right) v_{i n}}{c}\right]^{k}\right\}
$$

Where $P$ is the output power of Wind Tubine (WT) ; $v_{i n}, v_{r}$ and $v_{o}$ is The starting wind speed, the rating wind speed and cut-out wind speed of the (WT), respectively ; $k$ and $c$ are the shape coefficient and the scale parameter of WT respectively $; P_{r}$ is the power rating. Also $h=\left(v_{r} / v_{i n}\right)-1$;

The probability density function was put into the right side of the optimal scenario quantile equation (1), the simplified formula (6) is extracted after calculating.

$\int_{0}^{1}\left[f_{w}(P)\right]^{1 /(1+r)} d P=c_{3} \Gamma(a)\left[\Gamma\left(t_{2}, a\right)-\Gamma\left(t_{1}, a\right)\right]$

The probability density function put into the left side of the optimal scenario quantile equation (1), after calculating to obtain the simplified formula for (7):

$\int_{0}^{z_{s}}\left[f_{w}(P)\right]^{1 /(1+r)} d P=c_{3} \Gamma(a)\left[\Gamma\left(t_{2} z_{s}, a\right)-\Gamma\left(t_{1}, a\right)\right]$

According to equation (1) around the equation are equal, the optimal scenario quantile equation of wind power is as follows:

$$
c_{3} \Gamma(a)\left[\Gamma\left(t_{2} z_{s}, a\right)-\Gamma\left(t_{1}, a\right)\right]=\frac{2 s-1}{2 S} c_{3} \tau(a)\left[\Gamma\left(t_{2}, a\right)-\Gamma\left(t_{1}, a\right)\right]
$$

Where, $\Gamma(x, a) \Gamma(a)=\int_{0}^{a} x^{a-1} e^{-x} d x$ is the incomplete gamma function; $c_{1}=v_{i n} / c$; $c_{2}=\left(v_{i n} / c\right)\left(h / P_{r}\right) \quad ; \quad c_{3}=\left(k c_{2}\right)^{-r /(1+r)}(1+r)^{a} ; t_{1}=c_{1}^{k} /(1+r) \quad ; \quad t_{2}=\left(c_{1}+c_{2}\right)^{k} /(1+r) ;$ Generally, $r=2$.

By solving the equation (8), the optimal scenario quantile of wind power $z_{n}(n=1, \cdots, N)$ can be obtained, according to the scenario probability density function integral to obtain the probability corresponding quantile. In addition,

$$
\begin{aligned}
& p_{0}(P=0)=p\left(v<v_{i n}\right)+p\left(v>v_{o}\right)=1-\exp \left[-\left(\frac{v_{i n}}{c}\right)^{k}\right]+\exp \left[-\left(\frac{v_{o}}{c}\right)^{k}\right] \\
& p_{\mathrm{r}}\left(P=P_{\mathrm{r}}\right)=p\left(v_{r}<v<v_{o}\right)=-\exp \left[-\left(\frac{v_{o}}{c}\right)^{k}\right]+\exp \left[-\left(\frac{v_{r}}{c}\right)^{k}\right]
\end{aligned}
$$

The photovoltaic probability density functions are as follows ${ }^{[13]}$ : 


$$
f\left(P_{V}\right)=\frac{\left(\frac{P_{V}}{P_{\max }}\right)^{\alpha-1}\left(1-\frac{P_{V}}{P_{\max }}\right)^{\beta-1}}{\tau(\alpha) \tau(\beta)} \cdot \tau(\alpha+\beta)
$$

Where, $\alpha$ and $\beta$ are the parameters shape of Beta distribution function, respectively. $P_{\max }$ is the output power of photovoltaic array. $\tau$ is the Gamma function.

According equation (1), the optimal scenario quantile of $\mathrm{PV} z_{p}(p=1, \cdots, N)$ can be obtained as follows,

$\int_{0}^{z_{q}} t^{\mu-1}(1-t)^{\theta-1} d t=\frac{2 s-1}{2 S} \int_{0}^{1} t^{\mu-1}(1-t)^{\theta-1} d t$

Where, $t=\frac{P_{V}}{P_{\max }} \in(0,1) ; \mu=\frac{\alpha+r}{1+r}>0 ; \theta=\frac{\beta+r}{1+r}>0$.

According to the above-mentioned principles, the asymptotically optimal scenario quantile $z_{n}$ and $z_{p}$ of wind and photovoltaic can be obtained by equation (8) and (12), the optimal scenario probability corresponding the quantile can be obtained from equation (2-4).

\subsection{Multi-Scenario Tree Models of Wind-Photovoltaic-Load}

Assumed that $s w, s v, s l$ are the sample numbers of Wind power, Photovoltaic and Photovoltaic scenarios, respectively. Thus the number of scenarios can be expressed as,

$$
\begin{aligned}
& S=s w \times s v \times s l \\
& \operatorname{pr}(s)=p_{s w i} p_{s v j} p_{s l k} i \in s w, j \in s v, k \in s l, s \in S
\end{aligned}
$$

So multi-scenarios of Wind-Photovoltaic-Load model space set can be expressed as:

$$
\Omega=\left\{\operatorname{pr}(s),\left\{K_{1}(s), K_{2}(s), K_{3}(s)\right\}\right\}: s \in S
$$

\section{Multi-Objective Model Based on Asymptotically Optimal Scenario and Its Solution}

With installation of WTs and PVs in the distribution networks, any change in the power flow may change the voltage profile. Since the $\mathrm{X} / \mathrm{R}$ ratio of the distribution lines is small, the WT or PV has much impact on the voltage profile.

\subsection{Objective Functions}

As mentioned before, power systems are inherently stochastic due to uncertainties in both intermittent energy sources and load demands. Consequently, the multi-objective DGs allocation and sizing in distribution networks is a nonlinear optimization problem with both continuous and discrete parameters and variables. The objective functions and constraints can be formulated as follows.

\section{-Voltage deviations of the Buses}

Voltage deviation determines the difference between the voltages in nodes with respect to the nominal voltage. It is assumed that tap position of transformers and the values of capacitors change stepwise.

Min $f_{1}(X)=\frac{1}{N_{d}} \sum_{t=1}^{N_{d}} \sum_{i=1}^{N_{b u}}\left|\frac{V_{i}^{t}-V_{i}^{*}}{V_{i}^{*}}\right| p r_{i}$ 
Where $V_{i}^{*}$ is desired voltage of network at the ith bus, $V_{i}^{t}$ is actual voltage of network at node $i$ during time $t . N_{b u s}$ is number of buses. $N_{d}$ is number of load variation steps. $p r_{i}$ is the scenario probability index.

\section{-Total power losses}

The second objective is to minimize the total active power losses for the next day, which can be modeled as:

$\operatorname{Min} f_{2}(X)=\sum_{t=1}^{T} \sum_{i=1}^{N_{b r}}\left(R_{i} \cdot\left|I_{i}^{t}\right|^{2} \cdot h^{t}\right)$

Where, $\mathrm{N}_{\mathrm{br}}$ is the number of branches at hour $\mathrm{t}$.

\section{-Constraints}

In order to have an optimal plan while maintaining the security and operational conditions, the following constraints should be met:

\section{-Power balance}

$$
\begin{aligned}
& P_{G_{s i}}+K_{1}(s) P_{D G W_{i}}+K_{2}(s) P_{D G S_{i}}-K_{3}(s) P_{D_{i}}=\sum_{j=1}^{m} V_{s i} V_{s i} Y_{i j} \cos \left(\theta_{i j}+\delta_{s j}-\delta_{s i}\right) \forall i, s \\
& Q_{G_{s i}}-K_{3}(s) Q_{D_{i}}=\sum_{j=1}^{m} V_{s i} V_{s i} Y_{i j} \sin \left(\theta_{i j}+\delta_{s j}-\delta_{s i}\right) \forall i, s
\end{aligned}
$$

\section{-Active power of DGs limits}

$$
\begin{aligned}
& P_{P V \text {.min }} \leq P_{P V}^{t} \leq P_{P V \cdot \text { max }} \\
& P_{W T \text {.min }} \leq P_{W T}^{t} \leq P_{W T \text {.max }}
\end{aligned}
$$

- Tap of transformers

$$
\operatorname{Tap}_{i}^{\min }<\operatorname{Tap}_{i}^{t}<\operatorname{Tap}_{i}^{\max }
$$

\section{- Node voltage limits}

$$
V^{\min }<V_{i}<V^{\max } \forall i \in S
$$

\section{- Distribution line limits}

$$
\left|P_{i j}^{\text {Line }}\right|<P_{i j, \max }^{\text {Line }}
$$

\subsection{Hybrid HBMO-PSO Algorithm for Multi-Objective Optimization}

Multi-objective optimization is the process of optimization of different conflicting objective functions when all the constraints and limitations are observed simultaneously is called multi-objective optimization problem (MOP). The MOP can be described as ${ }^{[16]}$ :

$$
\begin{gathered}
\text { Min } F=\left[f_{1}(X), f_{2}(X), \ldots f_{n}(X)\right]^{T} \\
\text { s.t: }\left\{\begin{array}{cc}
g_{i}(X)<0 & \mathrm{i}=1,2 \ldots \text { Nueq } \\
h_{i}(X)=0 & \mathrm{i}=1,2 \ldots \text { Neq }
\end{array}\right.
\end{gathered}
$$

Where, $\mathrm{X}$ is the control variable of making decision. Also $\mathrm{n}$ is the number of objective functions. For a multi-objective optimization problem, two solutions $\mathrm{X}$ and $\mathrm{Y}$ can have one of these two possibilities: one dominates the other or none dominates the other. In a minimization problem, without loss of generality, a solution $\mathrm{X}$ dominates $\mathrm{Y}$ if the following two conditions are satisfied:

$\forall j \in\{1,2 \ldots n\} . f_{j}(X) \leq f_{j}(Y)$

$\exists k \in\{1,2 \ldots n\} \cdot f_{k}(X) \leq f_{k}(Y)$ 
3.2.1. The Original HBMO : Honey bee as a social insect with special behaviors and instructions has been the source of inspiration for the human beings during the years ${ }^{[17]}$. The honey bees' society is consisted of three groups in general: the queen or female, the drones or males and the workers. Each of these groups has a special task which should be implemented in such a way that the total condition of their society improves effectively. HBMO algorithm simulates each of the phases of the natural mating process so that to give a satisfying algorithm which would be profitable in the optimization applications. The mating process between the queen and each of the drones is implemented probabilistically with an annealing function as follows ${ }^{[18]}$ :

$\operatorname{prop}(D)=\exp \left(-\frac{\Delta f}{S(t)}\right)$

After each mating process, the queen speed decreases. If the mating process is successful, the corresponding drone sperm is added to the queen spermatheca, else it is discarded and the next drone is chosen for mating. The speed of the queen after each mating process is updated as follows:

$S(t+1)=\alpha \times S(t)$

The mating process continues until the time that the speed of the queen reaches to a specific value or her spermatheca become full. Now the breeding process is simulated. If the position of any of the new broods is better than that of the queen, then it will replace the queen. This process of mating and breeding continues until the time that the best satisfying queen (solution) would be achieved.

3.2.2. Hybrid HBMO - PSO Algorithm: The original HBMO suffers from two main deficiencies; that is the reliance of the HBMO algorithm on its parameters and the possibility of being trapped in local optima. These two shortcomings root from the mating process. Thus in order to improve the algorithm performance, the mating process should be corrected sufficiently. Player and motion speed is calculated through PSO relations in this method and choosing or suitability is obtained from HBMO method. Suitability analysis is performed using prob. Function in HBMO method. In (26), S(t) is equal to particle speed in PSO method and $\Delta f$ is showing difference from general optimized amount which can be calculated as below:

$\Delta f=\operatorname{gbest}(i t,:)-\operatorname{psot}(i)$

Where $\operatorname{gbest}(i t,:)$ is the best amount in all conditions and $p s o t(i)$ is the present position of the particle. The gbest(it,:) can be solution by PSO method. In PSO, the velocity $\mathrm{v}_{\mathrm{i}}$ and position $\mathrm{x}_{\mathrm{i}}$ of the dth dimension of the ith particle are updated as following:

$v_{i}^{t+1}=w v_{i}^{t}+\mu_{1} \cdot r_{1}\left(\right.$ pbest $\left._{i}-x_{i}\right)+\mu_{2} \cdot r_{2}\left(\right.$ gbest $\left.-x_{i}\right)$

$x_{i}^{t+1}=x_{i}^{t}+v_{i}^{t+1}$

Where pbest $_{i}$ is the best location in the search space ever visited by particle I; gbest is the best location discovered so far. The learning factors are $\mu_{1}=\mu_{2}=2 ; w$ is the inertia or momentum weight factor.

Now by the use of Eq.(25), the non-dominated solutions of the drones population are evaluated and stored in the repository.

3.2.3. Fuzzy-based Clustering: As mentioned before, the set of Pareto optimal solutions which are found during the optimization process are stored in an external memory (or repository). Since the repository size is constant, the number of the Pareto solutions should not exceed a specified number. Therefore a fuzzy-based clustering technique is 
utilized here to control the size of the repository. The membership function assigned to each objective function is as follows:

$\mu f_{i}(X)= \begin{cases}1 & f_{i}(X) \leq f_{i}^{\text {min }} \\ f_{i}^{\max }-f_{i}(X) & f_{i}^{\text {min }} \leq f_{i}(X) \leq f_{i}^{\max } \\ f_{i}^{\text {max }}-f_{i}{ }^{\text {min }} & f_{i}(X) \geq f_{i}^{\text {max }}\end{cases}$

The values of $f_{i}^{\text {min }}$ and $f_{i}^{\text {max }}$ are separately evaluated by single optimization of each objective function. Finally, for each of the solutions in the repository, the normalized membership function can be evaluated as follows:

$N_{\mu}(j)=\frac{\sum_{i=1}^{n} \omega_{i} \times \mu_{f l}\left(X_{j}\right)}{\sum_{j=1}^{m} \sum_{i=1}^{n} \omega_{i} \times \mu_{f l}\left(X_{j}\right)}$

Where $\mathrm{n}$ is the number of the objective functions and $\mathrm{m}$ is the number of the Pareto solutions in the repository. Therefore, after the evaluation of $N_{\mu}$ for all the Pareto solutions by Eq. (31), the repository is sorted in descending order. The best compromised solution is that for which the value of ${N_{\mu}}_{N}$ is maximum. Note that here $\omega_{i}$ is supposed to be unit so that to give equal preferences to all the objective functions. For multiple objective problems, the fuzzy solution can be calculated as: $\operatorname{Object}(X)=\min \left[\mu_{f 1}(X), \mu_{f 2}(X), \mu_{f 3}(X), \mu_{f 4}(X)\right]$, The maximum value of object $(\mathrm{X})$ is considered as the optimal solution.

\subsubsection{Implementing the Hybrid HBMO-PSO Algorithm for DG Placement}

This section presents the proposed hybrid algorithm based on the combination of HBMO and Pareto sets for multi-objective REGs placement. To apply the proposed algorithm in the REGs placement problem, the following steps have to be taken:

Step 1: defining the input data.

Step2: generation of the initial population. The initial population (Pop) is as follows:

$X_{i}=\left[\right.$ location $_{1}$, location $_{2}, \ldots$, location $\left._{n}, P_{1}, P_{2}, \ldots P_{n}\right]$

Pop $=\left[\begin{array}{c}X_{1} \\ X_{2} \\ \ldots \\ X_{N i}\end{array}\right]=\left[\begin{array}{cccc}F_{11} & F_{21} & F_{31} & F_{41} \\ F_{12} & F_{22} & F_{32} & F_{42} \\ \ldots & \ldots & \ldots & \ldots \\ F_{1 N} & F_{2 N} & F_{3 N} & F_{4 N}\end{array}\right]$

Step 3: $\mathrm{i}=1$

Step 4: select the ith drone. The values of the objective functions for the ith drone are evaluated using results of the distribution load flow.

Step 5: If the drone is a non-dominated solution, then the drone is stored into the repository and the fuzzy clustering is used to control the repository's size.

Step 6: if all drones are selected, go to Step 8, otherwise $\mathrm{i}=\mathrm{i}+1$ and return to Step 5.

Step 7: Select a queen (Xbest) from the repository randomly.

Step 8: Generate the queen speed.

Step 9: Select the population of the drones.

Step 10: Generate the queen's spermatheca matrix.

Step 11: Feed the selected broods and queen with the royal jelly by workers.

Step 12: The values of the objective functions for each brood are evaluated by using the results of the distribution load flow. If each brood is a non-dominated, then, store the brood into repository and use the fuzzy clustering to control its size, else the termination criteria should be checked. If the termination previous trial solutions then return to the Step 3 until convergence criteria is met. 


\section{Simulation Results}

\subsection{Optimal Scenarios Analysis}

To demonstrate the placement of DGs, IEEE 33-bus distribution test systems ${ }^{[19]}$ of Figure .1 were adopted to verify the proposed method.

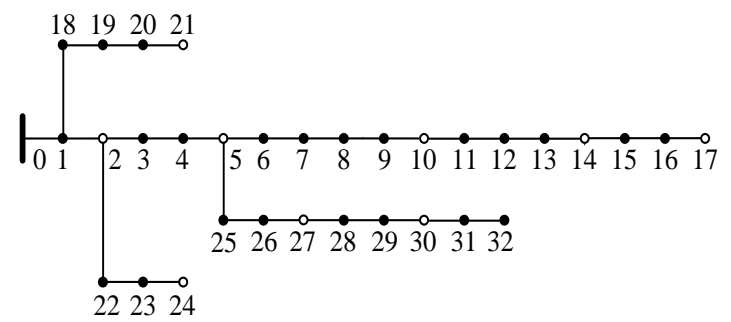

Figure 1. IEEE 33- Bus Distribution Test System

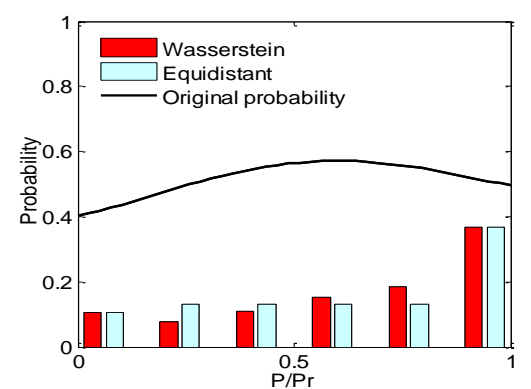

Figure 2. Comparison Diagram of Scene Simulation

To deal with the proposed stochastic programming models with multiple distributed generations (DG), firstly, the continuous probability density functions of wind power or photovoltaic generation were optimally transformed to discrete quantiles with precise probabilistic information based on Wasserstein distance metric. Then, with computational scales and loss of probability considered, the scheduling interval was reasonably divided into several parts. Hence, generate 10 asymptotically optimal scenarios for WT and PV, respectively. So the scenarios of Wind power, Photovoltaic and load are $s w=10, s v=10$, $s l=10$. The total number of scene space is $S=1000$. The relevant parameters of wind and PV model as shown in Table 1.

Table 1. Parameters of WT and PV model

\begin{tabular}{|c|c|c|c|c|c|c|c|c|}
\hline \multicolumn{4}{|c|}{ Parameters of WT } & \multicolumn{3}{c|}{ Parameters of PV } \\
\hline $\operatorname{Pr} / \mathrm{kW}$ & $v_{i}(\mathrm{~m} / \mathrm{s})$ & $v_{r} /(\mathrm{m} / \mathrm{s})$ & $v_{o}(\mathrm{~m} / \mathrm{s})$ & $K$ & $C$ & $\begin{array}{c}\text { photo-electric conversion } \\
\text { efficiency } \%\end{array}$ & $\alpha$ & $\beta$ \\
\hline 450 & 5 & 15 & 45 & 2 & 15 & 15 & 2.4 & 3.5 \\
\hline
\end{tabular}

In order to dedicate accuracy of the proposed model can represent the stochastic characteristics features of Renewable Energy Source (RES), e.g. wind power and photovoltaic (PV) power, cases are carried out in this paper. Firstly, to indicate the superiority of the proposed method, the real data of a distributed renewable energy generation demonstration project of Gansu in China is selected as sample to verify effectiveness of the asymptotically optimal scenarios. Figure. 2 show comparison results among original probability, Wasserstein distance and Equidistant method. It can be seen from Figure. 2 that the conventional scene methods (such as Equidistant method) are not similar to original probability distribution in a good way. Wasserstein distance method is obviously better than Equidistant method, because it can better reflect the original probability . The simulation results dedicate the advantages of Wasserstein distance used in the optimal scene analysis.

To further illustrate the asymptotically optimal scenarios based Wasserstein distance method can dedicate the stochastic characteristics features of the wind power and PV. Figure. 3 showed the 10 scenarios of WT (PV) output power comparing with daily historical data in spring. In Figure 3, the black line is curve of a wind power (PV power) 
daily historical data, while the rose line is the scenarios of WT (PV) output power by the proposed method. According to Figure 3, the volatility of scene distribution between random variables and the original distribution be approximate consistent, and the error between the two is minimum. The optimal scenes based on the Wasserstein distance indicators reflect the random feature of distributed generation active power output more accurately. These cases studies illustrate the precision and practicality of the asymptotically optimal scenarios based on Wasserstein distance metric.

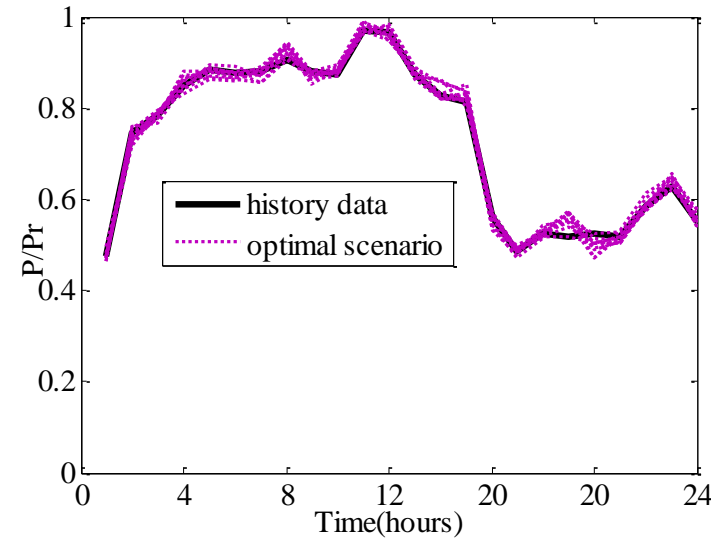

(a) Scenarios of WT output in spring

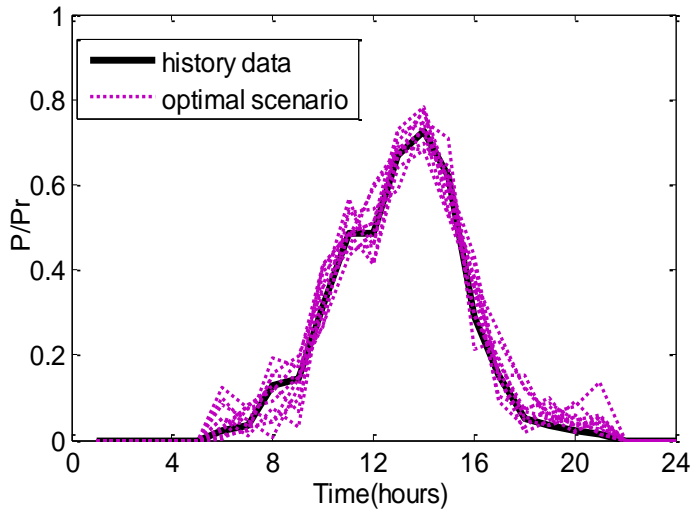

(b) Scenarios of PV output in spring

Figure 3. Comparison Diagram of Scenario with Historical Data

\subsection{Multi-Objective Optimized Results}

In this section, the hybrid HBMO-PSO algorithm is employed to solve the problem considering stochastic model of wind/PV and loads by the scenes. Based on the set of representative scenarios, the stochastic optimization problem is transformed into a deterministic optimization problem. There are several parameters to be determined for implementation of MHBMO algorithm. The best values for these parameters are selected as: $S_{\text {max }}=1, S_{\text {min }}=0.2, \alpha=0.92, N_{D}=20, N_{\text {Dron }}=N_{\text {Work }}=N_{\text {Brood }}=100, N_{\text {Sperm }}=15$, The number of the initial population is 100 .

Parameters of buses like bus voltage are obtained for sitting and sizing of DGs on mentioned distributed system, by implementation of the proposed algorithm. Figure 4 shows the convergence rate of the Improved HBMO algorithm in 20 trials. The best solutions obtained by optimizing $f_{l}(x)$ and $f_{2}(x)$ separately are 0.03397 (p.u) and $121.9 \mathrm{~kW}$. According to Figure 4, using the improved HBMO algorithm, the objective function reaches its minimum after about 120 iterations, and does not vary thereafter while the PSO algorithm converges to global optimum in about 145 iterations. So the PSO-HBMO algorithm has better outperforms the original HBMO. 


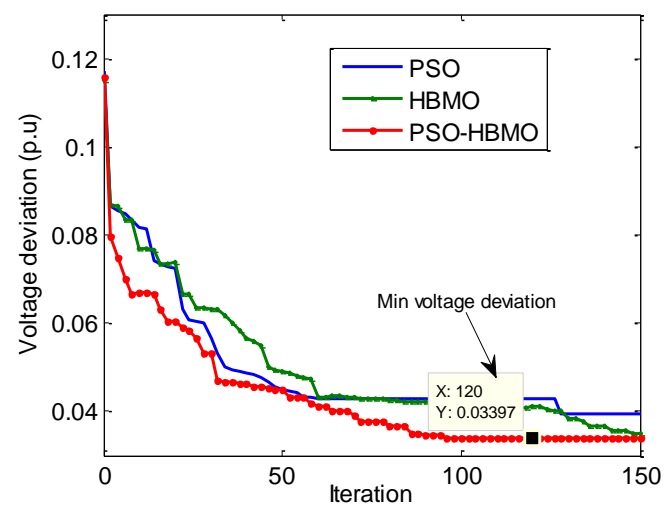

(a) The Best Solution for Voltage Deviations.

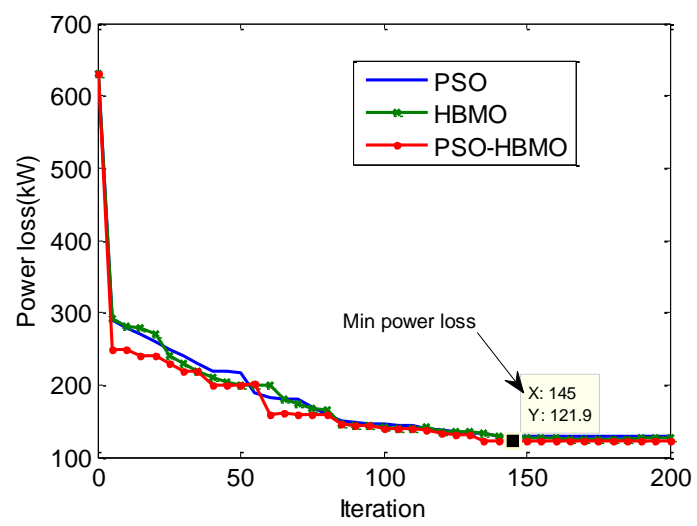

(b) The Best Solution for Power Loss

Figure 4. Convergence Characteristics of the Improved HBMO for the Best Solution

Here two cases are considered. In case I only one type DG installation is assumed. In case II two types DGs are assumed to be installed, DG sizes in the optimal locations, total real power losses before and after DG installation for two cases are given in Table 2. The best node place DG and its sizes are shown in Table 3.

Table 2. Result of Placement DGs on the Test System

\begin{tabular}{|c|c|c|c|c|c|c|}
\hline Algorithm & $\begin{array}{c}\text { Best } \\
\text { place }\end{array}$ & $\begin{array}{c}\text { Best } \\
\text { sizing } \\
\mathrm{kw}\end{array}$ & $\begin{array}{c}\text { Without } \\
\text { DG } \\
\text { loss(kw) }\end{array}$ & $\begin{array}{c}\text { With DG } \\
\text { loss(kw) }\end{array}$ & $\begin{array}{c}\text { Without DG } \\
\text { Min voltage } \\
\text { p.u }\end{array}$ & $\begin{array}{c}\text { With DG } \\
\text { Min voltage } \\
\text { p.u }\end{array}$ \\
\hline PSO & Bus 22 & 1872.7 & 255.00 & 128.9012 & 0.9378 & 0.9512 \\
\hline HBMO & Bus 22 & 1863.0 & 255.00 & 127.5177 & 0.9378 & 0.512 \\
\hline PSO-HBMO & Bus 22 & 1876.4 & 251.79 & 121.9001 & 0.9375 & 0.96003 \\
\hline
\end{tabular}

Table 3. Results of Test System

\begin{tabular}{|l|l|l|c|c|c|}
\hline $\begin{array}{l}\text { Test } \\
\text { system }\end{array}$ & Place method & \multicolumn{1}{|c|}{ DG location } & \multicolumn{1}{|c|}{$\begin{array}{c}\text { Best node (DG } \\
\text { sizing/kW) }\end{array}$} & $\begin{array}{c}\text { Voltage } \\
\text { margin /pu. }\end{array}$ & $\begin{array}{c}\text { voltage } \\
\text { /pu. }\end{array}$ \\
\hline $\begin{array}{l}\text { IEEE- } \\
33\end{array}$ & Without DG & \multicolumn{1}{|c|}{0} & 0 & - & 0.9378 \\
\cline { 2 - 6 } & With WT & $\begin{array}{l}5,10,14,17,21, \\
24,26,27,30\end{array}$ & $27(900)$ & 1.0351 & 0.9802 \\
\cline { 2 - 6 } & With PV & $\begin{array}{l}5,10,14,17,21, \\
24,26,27,30\end{array}$ & $27(800)$ & 1.0322 & 0.9876 \\
\cline { 2 - 6 } & With WT and PV & $\begin{array}{l}5,10,14,17,21, \\
24,26,27,30\end{array}$ & $\begin{array}{l}\text { WT in 27 (450) } \\
\text { PV in 27 (400) }\end{array}$ & 1.0341 & 0.9969 \\
\end{tabular}

The simulation results show that the reasonable choice of placement and sizing of distributed generation helps raise the voltage stability margin of distribution system and reduce the loss. The change in voltage profile with and without DGs on distribution test system can be observed in Figure 5. It can be observed from the Figure that the bus voltages are maintained within the permitted range of tolerance, i.e. $\pm 5 \%$ of the nominal value. 


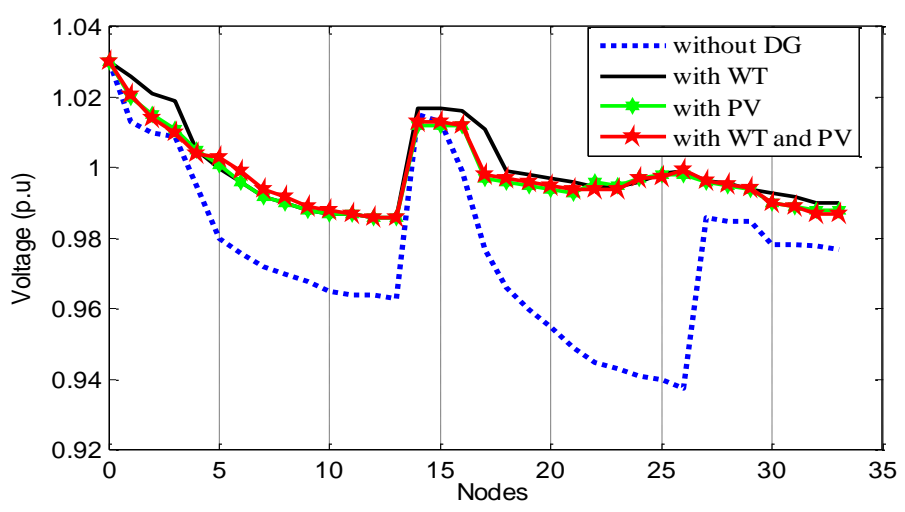

Figure 5. Voltage Distribution of 33-Bus System

\section{Conclusions}

In view of the uncertainties of intermittent distributed generation and load, a multiobjective model based on asymptotically optimal scenario for sitting and sizing of the DGs for distribution systems is presented. By using Wasserstein distance metric, the best approximation of the probability distribution of the Multi-scenario tree model of WindPhotovoltaic-Load was established. Then optimal scenes were generated from solving the model. A small number of optimal scene generated by using Wasserstein distance method, It can not only accurately simulate the random characteristics of distributed power, but also simplifies the calculation difficulty of the corresponding solving stochastic variable model. Moreover, the total electrical energy losses; the total emission and the grid and the voltage deviations of the bus were included in the objective function. A new optimization algorithm based on Hybrid HBMO-PSO algorithm was proposed to determine the strategy. In the proposed algorithm, a set of non-dominated solutions called Pareto-optimal solutions are found and stored in the repository which its size is controlled by the use of fuzzy clustering method. The simulation results show that the voltage magnitude of buses is in the desired limits. Best place and size of DGs were found at 33bus test system and two kinds of DGs with two kinds nominal capacities were compared to obvious the placement effect of them on distributed system. Results were showed accuracy of presented algorithm.

\section{Acknowledgements}

This work was supported by the National Natural Science Foundation of China (No.51467009), Science and Technology Foundation of STATE GRID Corporation of China (No. 271532-KJ-62); the project of Lanzhou science and technology plan (No.2014-1-162).

\section{References}

[1] A Kargarian Marvasti, Y Fu, S DorMohammadi, and M Rais-Rohani, Optimal Operation of Active Distribution Grids: A System of Systems Framework, IEEE Transaction on Smart Grid, vol. 5, no.3, ( 2014), pp. pp. 1228-1237.

[2] Liu T, Xu G, Cai P, Tian L, Huang Q. Development forecast of renewable energy power generation in China and its influence on the GHG control strategy of the country. Renewable Energy, Vol.36, No.4, (2011), pp. 1284-1292,

[3] M Zakaria Kamh, and R Iravan, Unbalanced Model and Power-Flow Analysis of Microgrids and Active Distribution Systems, IEEE Transaction on Power Delivery, vol. 25, no.4, (2010), pp.2851-2858.

[4] C.T. Borges, D.M. Falcao,, Optimal distributed generation allocation for reliability, losses, and voltage improvement, International Journal Electrical. Power Energy System, Vol.28, No.6, (2006), pp.413-420.

[5] V. H.Mendez, J.R.Abbad, andT. G. S. Roman, Assessment of energy distribution losses for increasing penetration of distributed generation, IEEE Trans. Power Syst., vol. 21, no. 2, (2006), pp. 533-540. 
[6] T. Gozel and M. H. Hocaoglu, An analytical method for the sizing and siting of distributed generators in radial systems, Electric Power Syst. Res., vol. 79,( 2009), pp. 912-918,

[7] Hong YY, Luo YF. Optimal VAR control considering wind farms using probabilistic load-flow and gray-based genetic algorithms. IEEE Transactions on Power Delivery, Vol. 25, No.4, (2010), pp. $2987-2996$,

[8] H Hedayati, S. A. Nabaviniaki, Adel. Akbarimajd, Amethod for placement of DG unit in Distribution network, IEEE. Trans. On Power Delivery Vol.21, No.8, (2007).

[9] H. Hedayati, S. A. Nabaviniaki, and A. Akbarimajd, A method for placement of DG units in distribution networks, IEEE Trans. Power Del., vol. 23, no. 3, pp. 1620-1628, 2008.

[10] F S. Abu-Mouti, M. E. El-Hawary, Optimal Distributed Generation Allocation and Sizing in Distribution Systems via Artificial Bee Colony Algorithm, IEEE Transaction on Power Delivery, Vol. 26, No. 4, vol. 23, no. 3, (2011), pp. 1520-1528.

[11] A Soroudi, Possibilistic-Scenario Model for DG Impact Assessment on Distribution Networks in an Uncertain Environment, IEEE Transactions on Power Systems, vol.27, no.3, (2012), pp.1283-1293.

[12] K Zou, A Prakash Agalgaonkar, K M. Muttaqi, and Sarath Perera, Distribution System Planning With Incorporation DG Reactive Capability and System Uncertainties, IEEE Transaction on Sustainable Energy, vol.3, no.1, (2012), pp.112-123.

[13] B Xiaoqing,W Xueying, L Peijie, Locating and Sizing of Photovoltaic Power Generations by Considering Voltage Stability Margin of Distribution System, modern electric power, vol.32,No.4,pp.34-41,2015.

[14] L Xian. Economic load dispatch constrained by wind power availability : a wait-and-see [14] approach[J]. IEEE Transactions on Smart Grid, vol. 1, no. 3, (2010), pp. 347-355.

[15] L Jinghua, W Hua, M Dong, Asymptotically Optimal Scenario Analysis and Wait-and-See Model for Optimal Power Flow With Wind Power[J], Proceedings of the CSEE, Vol.32 No.22, (2012), pp. 15-23.

[16] T Niknam, B Firouzi B, Ostadi A. A new fuzzy adaptive particle swarm optimization for daily Volt/Var control in distribution networks considering distributed generators. Applied Energy, Vol.87, No.6, (2010), pp.1919-1928.

[17] Afshar, A., O. Bozog Haddad, M.A. Marino and B.J. Adams, Honey bee mating optimization (HBMO) algorithm for optimal reservoir operation, Journal of the Franklin Institute", Vol.344, No.5, (2007), pp.452-462 .

[18] M Fathian, B Amiri, A Maroosi. Application of honey bee mating optimization algorithm on clustering, Applied Mathematics and Computation, Vol.190, No.2, (2007), pp.1502-1513,

[19] Voltage stability-based DG placement in distribution network, IEEE Transaction on power deliver, Vol.28, No.1, (2013), pp. 171-178.

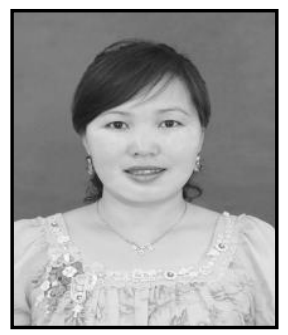

Lizhen Wu. She received the M.S. degree in the control theory and control engineering from Lanzhou University of Technology, Gansu, China, in 2004. And now she is studying for her doctorate in power system\& its automation. Currently, she is an Associate Professor at College of Electrical and Information Engineering Lanzhou University of Technology, where she teaches courses on power electronics, control theory, and renewable energy systems. Her interests include Photovoltaic, wind energy conversion, storage energy systems, and micro-grids; power system control and distribute generation sources connected to the distribution network. 